\title{
Use of Mixed Stationary Phases for the Separation of Metal Ions
}

\author{
I. V. Pletnev†, E. I. Morosanova and Yu. A. Zolotov \\ Chemistry Department, M. V. Lomonosov Moscow State University, Lenin Hills, 119899, Moscow, USSR
}

\begin{abstract}
The theoretical foundations and applications of "mixed" adsorbents for the separation and concentration of metal ions are considered. "Mixed" adsorbents are defined as those containing two or more kinds of functional groups (with different donor sets) in a controlled proportion. Varying the proportion allows one to easily regulate the affinity of the stationary phase to metals. Experiments were carried out with the use of reversed-phase thin-layer chromatography (silica plates were impregnated with solutions of macrocyclic compounds possessing different $\mathrm{N}_{x} \mathrm{O}_{y}$ donor sets in a nonpolar solvent). "Mixed" stationary phases were shown to be capable of providing the strongest retention for metals which are not retained most strongly on any of the parent individual adsorbents. Also possible is an improvement in the separation when the use of individual phases leads to overlapping.
\end{abstract}

Keywords Metal ion separation, metal ion concentration, mixed stationary phase

Modern inorganic trace analysis required in adsorption materials allows one to separate and concentrate metal ions. Especially important are those suitable for easily automated flow technique. ${ }^{1}$ Typically, such adsorbents should be capable of binding strongly a certain metals; others are eluting. This requires a capability to design adsorbents suitable for special tasks with definite affinity to metal ions. The present study was devoted to finding a general way to achieve this goal, by using "mixed" adsorbents.

\section{Experimental}

Stock solutions of metal nitrates (chemically pure) were prepared by dissolving accurately weighed amounts in distilled water. Macrocycle diaza-18-crown6 (DA18C6, see Fig. 1) was obtained from the Cherkassky plant of chemical reactives, USSR; macrocycles diazadibenzo-18-crown-5 (DADB18C5) and tetraazadibenzo18-crown-6 (TADB18C6) were synthesized by Dr. A. A. Formanovsky, Vernadsky Institute of Geochemistry and Analytical Chemistry, Moscow, USSR. Solutions of macrocyclic compounds were prepared by accurately dissolving weighed amounts in a mixture of chloroform and heptane $(1: 1)$.

Thin-layer chromatography (TLC) silica-plastes (Silufol, Czecho-Slovakia) were dried at $120^{\circ} \mathrm{C}$. After that these plates were modified by dipping into either a $5 \times 10^{-3} \mathrm{M}$ solution of a chosen macrocycle or a mixture of two compounds with the same total concentration.

A mobile phase ( $\mathrm{pH} 5.8$ ) was used which contained a $0.02 \mathrm{M}$ acetate buffer solution with the addition of

\footnotetext{
$\dagger$ To whom corrsepondence should be addressed.
}

sodium perchlorate $(0.05 \mathrm{M})$. It was previously shown that macrocycles are retained well on TLC-plates, while forming an uniformly distributed layer and are not eluted by either water or acetate buffer solutions. Metal solution samples were $4 \mathrm{ml}$ in volume at a concentration of $5 \times 10^{-4} \mathrm{M}$. The location of metals was detected visually after drying and development with appropriate reagents $(1 \%$ solution of rubeanic acid in ethanol for $\mathrm{Ni}$ and $\mathrm{Cu}, 5 \%$ solution of 8-quinolinol in ethanol for $\mathrm{Zn}$, water solution of $\mathrm{KI}$ for $\mathrm{Pb}$, acetone/ water solution of Orthanylic $\mathrm{C}$ for $\mathrm{Ba}$ ).

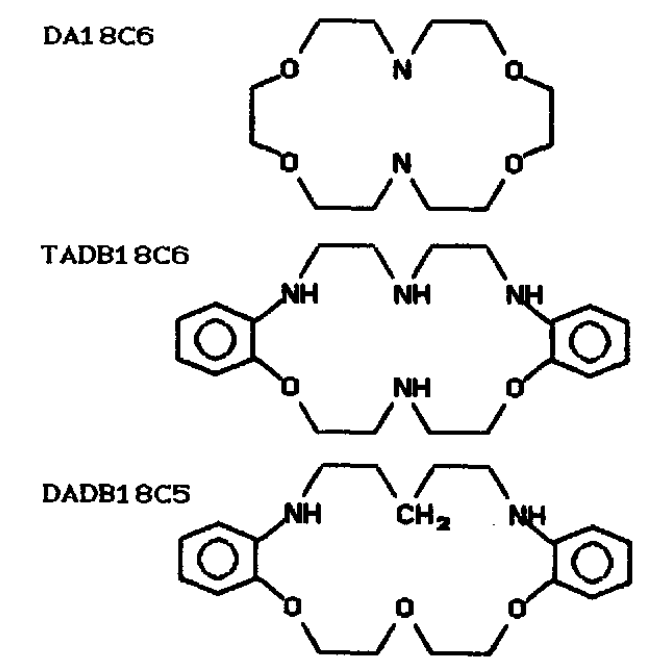

Fig. 1 Compounds used for the modification of TLC plates. 


\section{Results and Discussion}

\section{"Mixed" adsorbents: theoretical overview}

Despite the various properties of adsorbents for the separation and concentration of metals, there seems to be one main feature responsible for their affinity to metal ions, namely the nature of the functional groups. By varying the kinds and number of heteroatoms one can vary the affinity (retention) order and separation ability.

Considerable efforts have been made to synthesize adsorbents bearing (covalent linked or impregnated) organic moieties capable of forming complexes with metals. The synthetic route usually includes as synthesis of appropriate free organic molecules followed by some procedure for their attachment to a polymeric support. This approach, however, has some limitations, both practical and theoretical.

From a theoretical point of view any metal ion can be characterized by its softness/hardness in terms of well-known Pearson concept. Any given metal ion must in the most strongest way bind to a sorbent with a functional group possessing a corresponding softness (soft metal-soft functional group, hard metal-hard group and so on).

It is not a very great challenge to find absorbents with great affinity to metals possessing distinct features, soft or hard ("soft" S-containing functional groups are known to be most suitable for the binding of "soft" mercury and silver). As to the metals with intermediate properties, it would be much more difficult to select the proper functional group of a sorbent.

The most obvious way is to combine soft and hard centers in a functional group. However, not every combination of complexing atoms can be easily realized "in a single molecule", synthetic procedures are often both time-consuming and complex. Moreover, some combinations can not be realized at all. For example consider an organic molecule with a ratio of soft (A) to hard (B) centers of 10 . Any attempt to create the donor set $A_{10} B$ would most likely result in such a situation that not all atoms would be included in the complexation; this means that the actual donor set and affinity to metal would be quite different from those suggested. Further, it is impossible to such systems as $A B_{0.1}$.

A very simple way seems to exist to overcome these difficulties, while allowing practically any affinity order to certain metals, involving the use of "mixed" adsorbents. By mixing two (or more) adsorbents bearing different functional groups or by "mixing" groups on one support we can produce an adsorbent with a "mixed", intermediate retention order of metals. By varying the fractions of the constituents in a "mixture" one can vary the affinity to any metal within the limits defined by the properties of pure, individual adsobents (groups).

Let's consider the mixture of two constituents, $L_{1}$ and $\mathrm{L}_{2}$. The distribution ratio of the metal, $D$, could be (assuming that $\left[\mathrm{L}_{1}\right]$ and $\left[\mathrm{L}_{2}\right]$ would be displaced for each separation plate by totals, $C_{1}$ and $C_{2}$ ) equal to $K_{1} C_{1}+K_{2} C_{2}$, where $K_{1}, K_{2}$ are constants of $\mathrm{M}-\mathrm{L}$ interactions. If the overall capacity, $C=C_{1}+C_{2}$, is constant ("mixing" means an equivalent displacement of $\mathrm{L}_{1}$ to $\mathrm{L}_{2}$ (or vice versa)), then $D=\left(\kappa_{1} K_{1}+\kappa_{2} K_{2}\right) C$. Here, $\kappa_{1,2}$ represents the fraction of ligands in the "mixture". By comparing with $D$ regarding the individual adsorbents of that capacity $\left(D_{1}=K_{1} C, D_{2}=\right.$ $K_{2} C$ ), we can express $D$ as $\kappa_{1} D_{1}+\kappa_{2} D_{2}$. Due to the proportionality between the retention and distribution ratio, a similar relation should also be valid for the retention. The retention of any metal on a "mixed" adsorbent is, thus, intermediate between the values for individual stationary phases; the retention is linearly proportional to the mixture composition (this is schematically shown in Fig. 2). In other words a "mixed" stationary phase must behave as a individual one having functional groups $\left[\left(\mathrm{L}_{1}\right)_{k 1}\left(\mathrm{~L}_{2}\right)_{\kappa 2}\right]$ (note that this is true at any $\kappa_{1}, \kappa_{2}$ values, even if they are fractional).

The most interesting point is that the mixed adsorbent may have the greatest affinity to a metal which is not bound most strongly on any parent individual adsorbent. If, for example, $L_{1}$ is a soft ligand, and $L_{2}$ is a hard one, there may exist a range of mixture compositions which would allow a strongest binding of neither $M_{1}$ (soft) nor $M_{2}$ (hard), but of $M_{3}$ having medium softness (region II on Fig. 2 (a)). It provides a very simple way to regulate the separation ability and to create adsorbents for special tasks.

Somewhat less interesting is the potential ability to use "mixed" adsorbents to enhance the separation of
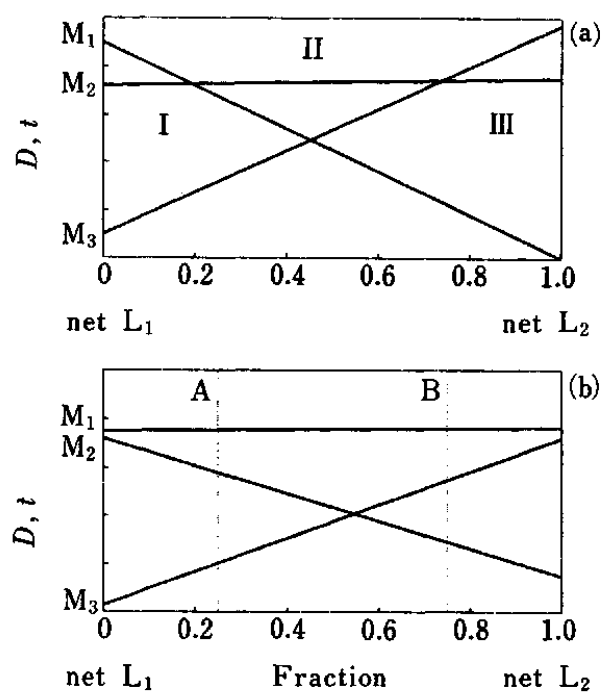

Fig. 2 Use of "mixed"adsorbents. (a) Change of the retention order: region $\mathrm{I}-\mathrm{M}_{1}>\mathrm{M}_{2}>\mathrm{M}_{3}$ (as for $\mathrm{L}_{1}$ alone); region III$M_{3}>M_{2}>M_{1}$ (as for $L_{2}$ alone); region II $-M_{2}>M_{1}, M_{3}$. (b) Improvement of separation. The use of individual phases results in poor separation; "mixtures" A, B show good separation. 
coeluting ions (Fig. 2(b)). Of the three metals shown in the scheme, one pair is not separated on the $\mathrm{L}_{1}$-adsorbent, and another is not separated on the $L_{2}$-phase. The "mixtures" corresponding to lines A and B provide a reasonable separation of all ions. This is evidently important for a chromatographic analysis.

\section{"Mixed" adsorbents: experimental results}

We unexpectedly found practically no mention regarding the use of "mixed" adsorbents in the literature concerned with the recovery and separation of metals, though we did find some examples in gas chromatography ${ }^{2}$ and high performance liquid chromatography ${ }^{3}$ of organic substances, confirming the validity of the considered approach. There has been only one recently published paper ${ }^{4}$ concerning the partial use of the "mixed" phase in inorganic chromatography. Unfortunately, this work was limited by the use of crownethers as "functional groups" (substances with one kind of donor atom are not very good choice for "mixing") and single "mixture" without any variation of composition; further, applications deal only with improvements in two-peak resolution.

Due to this lack of data we have carried out experiments examining the usefulness of "mixed" adsorbents approach.

Reversed-phase TLC was used as a simple, fast chromatographic method which allows to use the "mixed" phases prepared by simple impregnating a plate with mixtures of organic reagents. In order to avoid any undesirable formation of mixed complexes (the schemes and formulas mentioned above may be written in this simple form only in their absence) macrocyclic compounds were used as constituents of mixtures. They are known to form such complexes only relatively rarely.

The main result is that "mixed" stationary phases really do behave as was believed (cf. Fig. 2(a) with Fig. 3(a), and Fig. 2(b) with Fig. 3(b)). Even though the correspondence between theoretical and experimental schemes is somewhat approximate, trends are evident. And though some lines in plots of $R_{\mathrm{f}} v s$. mixture compositions (for $\mathrm{Ni}$ of Fig. 3(a); for $\mathrm{Cu}$ and $\mathrm{Pb}$ on Fig. 3(b)) are not straight, they have a suggested shape. First of all, the differences may be explained by the fact that the compositions given in Figs. 2 and 3 are not true compositions of mixtures existing on the silica surface (which is very difficult to determine). This is simply the composition of a solution used for the modification of plates. There is undoubtedly a proportionality between these values; however, there may be no linear relationship. Another possible reason regards a number of assumptions made through theoretical considerations.

Consider Fig. 3(a). The use of an individual "harder" modificator (donor set $\mathrm{N}_{2} \mathrm{O}_{4}$; diaza-crowns are known to be similar to "hard" crown-ethers) results in the retention order $\mathrm{Zn}>\mathrm{Pb}>\mathrm{Ni}$ (corresponding to the decrease in metal hardness); the use of a "softer" $\left(\mathrm{N}_{4} \mathrm{O}_{2}\right)$ modificator alone results in a fully inverse series. The
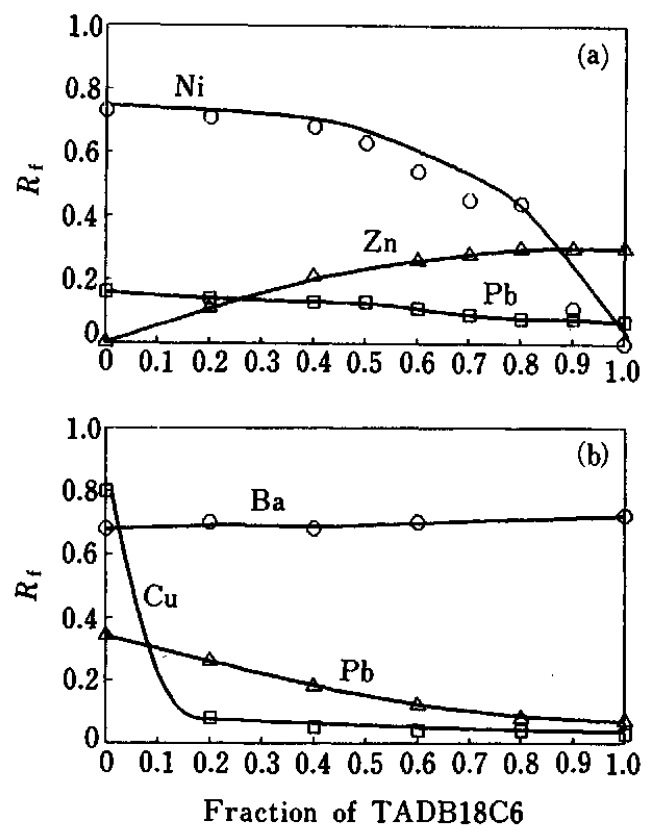

Fig. 3 Retention of metals on TLC plates impregnated with mixtures of TADB 18 C6 with DA18C6 (a) or DADB18C5 (b). Note that the picture is inverted relative to Fig. 2 due to the use of $R_{\mathrm{f}}$ instead of $D, t$.

use of some mixtures (intermediate softness) results in a maximal retention of $\mathrm{Pb}$ having "intermediate" properties and not being retained by both the individual sorbents in the strongest way.

Though the example shown in Fig. 3(b) is not so prominent and clear, it does demonstrate the ability of "mixed" adsorbents to improve chromatographic separation. Neither modification by $\mathrm{N}_{2} \mathrm{O}_{3}$ - nor by $\mathrm{N}_{4} \mathrm{O}_{2}$ macrocycle provides any reasonable separatin of $\mathrm{Ba}, \mathrm{Cu}$ and $\mathrm{Pb}$. However, due to a difference in overlapping pairs for the individual adsorbents, some "mixtures" make it possible.

The use of "mixed" adsorbents for column separation is probably the most interesting application. It can be made by either mixing of impregnating reagents, or a simple mechanical mixing of adsorbents with different chemically linked groups. This work is now in progress.

The authors are very grateful to Prof. N. M. Kuz'min for stimulating discussions and to I. M. Maximova for experimental assistance.

\section{References}

1. Yu. A. Zolotov and N. M. Kuz'min, "Preconcentration of trace elements", Elsevier, Amsterdam, 1990.

2. E. Fernandez-Sanchez, J. A. Gracia-Dominguez, V. Menendez and G. Del Rio, Anales de Quimica, 82, 507 (1987). 
3. J. B. Growther, S. D. Fazio and R. A. Hartwick, J. Chromatogr., 282, 619 (1983).

4. K. Kimura, H. Harino, E. Hayata and T. Shono, Anal. Chem., 58, 2233 (1986).

(Received March 31, 1990) (Accepted July 7, 1990) 